\title{
Building Detection using Local Gabor Feature
}

\author{
Zaaj Ibtissam, PhD \\ Laboratory of Industrial and \\ Computer Engineering \\ National School of Applied \\ Sciences, Ibn Zohr University \\ Agadir, Morocco
}

\author{
Chaouki Brahim El khalil \\ Prof, Laboratory of Industrial and \\ Computer Engineering \\ National School of Applied \\ Sciences, Ibn Zohr University \\ Agadir, Morocco
}

\author{
Masmoudi lhoussaine \\ Prof, Geomat Laboratory, Physics \\ Dept \\ Faculty of Science, Mohammed V \\ University \\ Rabat, Morocco
}

\begin{abstract}
Several approaches are based on the extraction of building contours by applying the Canny filter. The latter uses the local first-order operator (gradient technique), followed by a search for local maxima. However, these techniques often give unsatisfactory results on satellite images where intensity changes are rarely sharp. These techniques also require a thresholding operation for better contour detection, which makes the automation of the approach very complicated. The alternative approaches to apply the Gabor filter which has the advantage of being located in space and in the frequencies, and very widespread because of its property of optimal joint resolution in frequency and time. Their use makes it possible to extract the contours of the images to characterize their texture. The final step is to group the pixels into a number of classes representing the texture regions. The k-means classification algorithm has been applied for this sake.
\end{abstract}

\section{Keywords}

Building Detection, Gabor Filter.

\section{INTRODUCTION}

We ask that authors follow some simple guidelines. The identification and extraction of objects from remote sensing data is a scientific challenge in the fields of image processing and artificial intelligence and remain increasingly used for the updating of spatial databases taking into account the availability of satellite images with different resolutions. Among all objects, roads and buildings constitute the basic structure of urban databases and are the subject of special attention. This work focuses on the extraction of buildings. Many methods are proposed in the literature. Some methods are presented according to extracted primitives (contours, lines, regions, texture ...).

Both the Gabor filter and the gradient feature are considered a fundamental operation in signal and image processing, and have provided the best results. They both have some common properties: they are applicable to both binary images and gray-scale images, and are immune to image noise; but the gradient feature with canny meets the threshold automation problem. A low threshold gives a lot of details and a high threshold gives less detail which produces an edge detection that is not correct; that's why we used the Gabor filter to avoid this problem.

Gabor filter is a band-pass filter selective to both orientation and spatial frequency. It is suitable for detecting local structural patterns from images, and has been widely applied to texture analysis and object recognition. Consequently, Gabor-filter-based methods have been successfully applied for a variety of machine vision applications [1], such as edge detection, object detection [2], texture segmentation [3], image classification, fingerprint and face recognition. Much research has been done with Gabor Filter; [4] Gabor filter performance in image segmentation becomes worse [5] to detect micro texture using Local Binary Patterns.

In [6] we propose a principled method for implementing Gabor filters for feature extraction. Based on the Gabor wavelet framework and the sampling theorem. The filter parameters are automatically determined from the scaling factor and the sampling interval. As to the selection of filter outputs, we observed that the magnitude performs much better than the real part, and the phase information does not help.

In this paper, a principle method for implementing Gabor filters for feature extraction has been proposed.

\section{PROPOSED APPROACH}

In this work, we propose a method of extraction of buildings from satellite images with very high spatial resolution. The method starts with a pre-treatment with entropy to eliminate small noisy objects considered, knowing that the objects tobe extracted usually do not take small sizes (buildings). For reliable contour detection, a spatio-frequency analysis is applied using a linear filter whose impulse response is a sinusoid modulated by a Gaussian function called Gabor's wavelet. Finally, the k-means algorithm makes it possible to provide a solution to image segmentation, and subsequently reliable identification of buildings. The following diagram shows the steps of our approach:

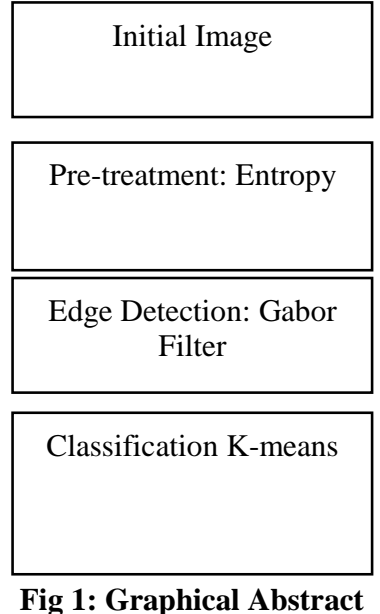

\subsection{Pretreatement by Entropy}

The application of the entropy on the image gives the entropic value of each pixel; it measures the amount of information contained or delivered by an information source. Our goal is the elimination of noise while preserving the contours by simply discarding small entropic values that have no meaning.

\subsection{Gabor Filter}

The performance of a detector is deeply related to the computation time and the efficiency of the detection. The effectiveness of detectors can be defined according to the following three criteria (Canny, 1986): 
- Good detection where all the contours must be detected without omitting certain pixels on the contour to be detected.

- Good location where the contours detected must be in their ideal position.

- Deleting Multiple Responses where a detector must not provide multiple responses or false contours.

The detection of contours by Canny does not always bring satisfactory results because its application does not allow separating the edge of the shells from the bottom of the image without causing false alarms. Moreover, the fact that it is not automatable presents a major constraint for the user (to have the expected result).

In effect, the user must fix an empirical threshold. Indeed, when decreasing the threshold one obtains a more detailed outline. On the contrary, any increase in the threshold will cause a decrease in the details in the result.

Since contour detection with Canny largely depends on the choice of threshold. This constraint makes it very difficult to automate the threshold. The use of the Gabor filter provides a potential alternative to Canny's contour detection. Our approach is to use this filter with specific orientations to the problems of building detection. After eliminating the noise found in the image, we are interested in the extraction step, which aims to determine the representative vector of the image using the Gabor filters. A Gabor filter is a sinusoidal function modulated by a Gaussian envelope. The sinusoidal function is characterized by its frequency and its orientation. Thus a Gabor filter can be seen as a detector of particular orientation edges, since it reacts to the edges perpendicular to the direction of propagation of the sinus. Gabor filtering preserves the temporal and frequency aspects of the signal. In the space domain, the application of Gabor filters is performed by calculating the convolution of the image with a function set to one of the textures. The process is shown in the following equation:

$$
\begin{aligned}
& q(x, y)=p(x, y) \times h(x, y) \\
& q(x, y) \text { Is the filtered pixel of the result image. } \\
& h(x, y) \text { is the Gabor filter. } \\
& p(x, y) \text { is the pixel of the real image. }
\end{aligned}
$$

The Gabor filters, used as they are, make it possible to obtain an efficient segmentation of an area of the image but provided that the exact scale of the object to be recognized is known: What will be the orientation $\theta\left(0^{\circ}, 30^{\circ}, 60^{\circ}, 90^{\circ}, 120^{\circ}, 150^{\circ}\right)$ and frequencies that will provide good contour detection.

\subsection{Choosing filter settings}

The objective of this study is to extract the buildings from the images. Given that buildings generally have well-defined geometric shapes (square or rectangle), the Gabor filter can be used with well-defined orientations. We assume that the buildings have small curvatures and that they can be represented by rectangles, their edges can be broken down into straight segments. It is therefore sufficient to take the two orientations $\theta=0$ and $\theta=90$, which will allow to greatly reduce the calculation time while eliminating the details that do not correspond to our problem. The image can be seen as a multi-frequency composition (several textures each of which is described by a specific frequency), and the filtering makes it possible to select only those components of the image whose frequency corresponds to that of the filter.

In our approach, several frequency values have been tested in order to find the right value with the processed image. To test the impact of choice of frequency, a series of tests have been performed designating the frequency by simple numbers ( $\mathrm{f}=$ $1.75, \mathrm{f}=2.74$ and $\mathrm{f}=4$ ). After applying the Gabor filter for contour detection in a satellite image, a classification step is required to isolate buildings, fields, roads and agricultural parcels.

\subsection{Classification by K-means}

Classification is the last step in this treatment chain. Indeed, there are many classification techniques in the literature: $\mathrm{k}$ means, EM method, Fuzzy c-means, Bayesian method, SVM, ISODATA, etc. For better achievement, methods with different characteristics have been chosen. By way of example, thanks to the k-means rapid convergence and their unsupervised mode, the Gaussian mixtures have been adapted to descriptors having a probability density close to a Gaussian. The main idea of the k-means algorithm is to choose randomly a set of centers fixed a priori and to search iteratively for the optimal partition. Each pixel is assigned to the nearest center, it constitutes the new class representatives, when they have reached a stationary state (no data changes class) the algorithm is stopped. The number of classes was estimated at 4 classes according to the components of the image: buildings, road, agricultural parcel and fields. Each class is defined by a number of characteristic vectors.

The main steps of k-means are:

- the initial position of $\mathrm{k}$ clusters (in this case $\mathrm{k}=4$ ).

- (Re-) assigning objects to a cluster according to a criterion of minimizing distances. Once all the objects have been placed, recalculated the $\mathrm{K}$ cancroids.

- Reapplying steps 2 and 3 until no more reassignments are made.

\section{RESULTS \& DISCUSSION}

The figures below display the results obtained after the application of Gabor filter on satellite image of QUICKBIRD nature.

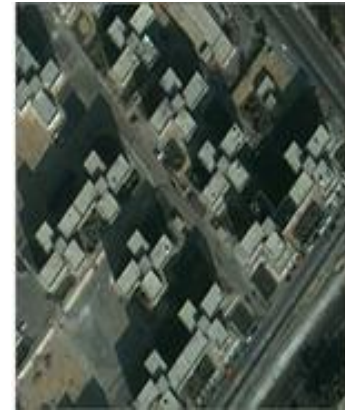

Fig2. Initial image

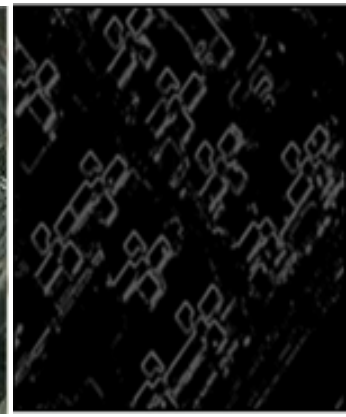

Fig3. Entropy image
Figure 3 shows the entropy value of each pixel that existed in the initial image.

To test the impact of choice of frequency, a series of tests have been performed designating the frequency by simple numbers ( $f=1.75, f=2.74$ and $f=4$ ). The figures below show the results of the Gabor filter application with different values of the frequency and two orientations $\theta=0$ and $\theta=90$. 

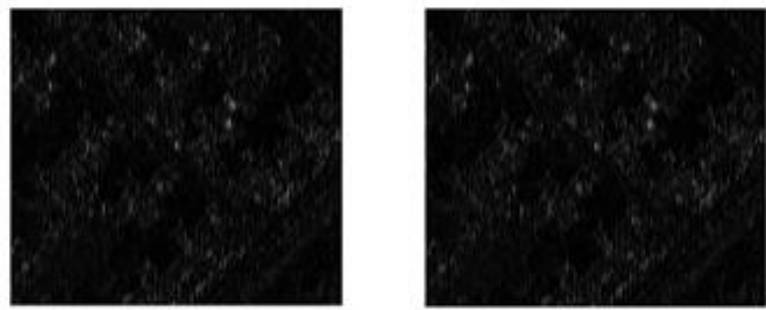

Fig 4:

$\theta=0 \mathrm{f}=2.74$ (gauche): $\theta=90 \mathrm{f}=2.74$ (droite)
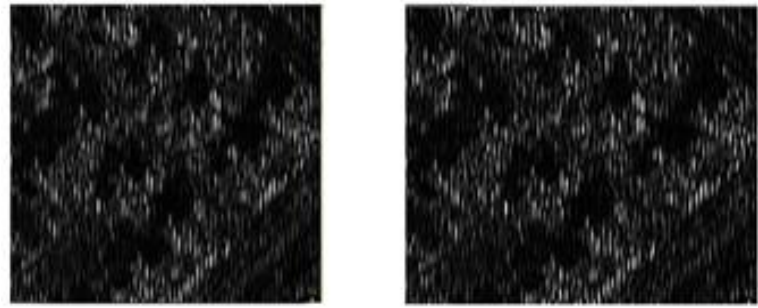

Fig 5: $\theta=0 \mathrm{f}=4$ (left): $\theta=90 \mathrm{f}=4$ (right)
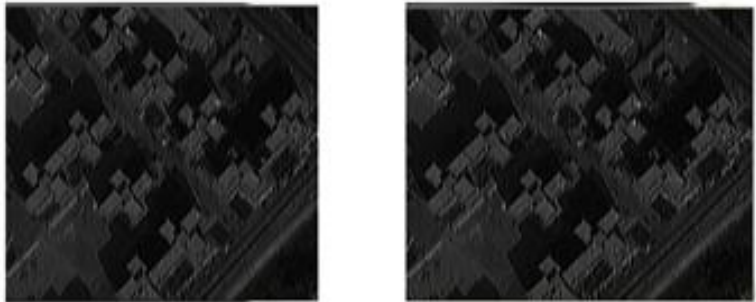

Fig 6: $\theta=0 \mathrm{f}=1.75$ (left): $\theta=90 \mathrm{f}=1.75$ (right)

According to the results obtained after application of Gabor filter on Quickbord satellite image, with two orientations $\theta=0$ and $\theta=90$ and with frequencies respectively $\mathrm{f}=1.75 \mathrm{f}=2.74$ $\mathrm{f}=4$. It can be seen that the outlines are well identified, but they are not really accurate.

Indeed, we notice that the processed image becomes a little blurred due to the increase in frequency.

On the contrary, with the decrease in the number of frequencies, more details are obtained, as can be seen in Fig. 6 that it has an almost global detection of all the objects that exist in the image.

The frequencies of the homogeneous zones are relatively low compared to the frequencies of the textured zones (high frequencies).

We can confirm the importance and completeness of the two main parameters of a Gabor filter: frequency and orientation. However, in this case of satellite images, these two parameters play a very important role in the filter response and the accuracy of the results.
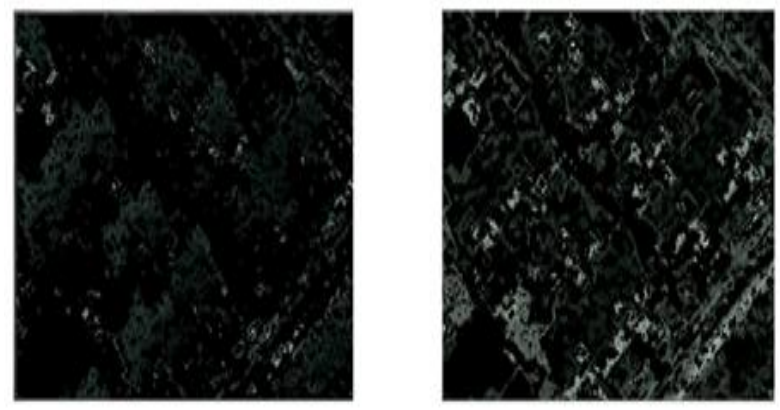

Fig 7: $K=1, K=2$
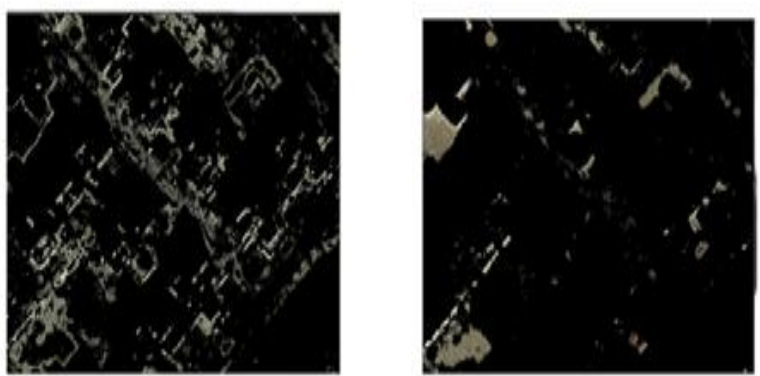

Fig 8: $K=3, K=4$

The results obtained by the k-means classification are acceptable, the algorithm arrives at classifying the existing classes in the image filtered by Gabor.

\section{CONCLUSION}

We presented a method for extracting buildings from satellite images with very high spatial resolution. This method is based on a spatio-frequency analysis by applying the Gabor filters followed by a classification with the k-means algorithm. The first results obtained are very encouraging and indicate that we can still improve them. The model can be adapted to other applications such as texture analysis, extraction of feature points or image indexing by content. As a conclusion, we think that Gabor filters are excellent extractors not only for buildings, but also for other types of textures. They have an optimal spatial / frequency joint resolution, which translates into correct detection. They are also quite easy to create and manipulate, due to its power making them a very effective tool in image segmentation.

\section{REFERENCES}

[1] T.S. Lee, IEEE Trans. Pat. Anal. Mach. Intell. 18, 959 (1996).

[2] A.K. Jaın, T. Nalını, K. Ratha, S. Lakshmanan, Pattern Recogn. 30, 295 (1997).

[3] U. Marmol, Arch. Photogram. Cartogr. Remote Sens. 22, 325 (2011)

[4] Li, M., \& Staunton, R. C. (2008). Optimum Gabor filter design and local binary patterns for texture segmentation. Pattern Recognition Letters, vol. 29, no. 5, 664-672.

[5] Mäenpää, T., \& Pietikäinen, M. (2006). Texture Analysis With Local Binary Patterns. In C. H. Chen, \& P. S. Wang, Handbook Of Pattern Recognition And Computer Vision, 3rd ed. (pp. 197-216). Singapore: World Scientific Publishing Co. Pte. Ltd.

[6] Cheng-Lin Liu, Masashi Koga, and Hiromichi Fujisawa 2005

[7] [Gabor, D.1946]. Theory of communication.Journal of 
International Journal of Computer Applications (0975 - 8887)

Volume 181 - No. 33, December 2018

the Institution of Electrical Engineers-Part III : Radio and Communication Engineering, 93(26), 429-441.

[8] [K. Jain.2000], S. Prabhakar, L. Hong and S. Pankanti, "Filterbank-based fingerprint matching", IEEE Transactions on Image Processing, vol. 9, no. 5, pp. 846859,2000

[9] [U. Marmol.2011], Arch. Photogram. Cartogr.Remote Sens. 22, 325 (2011).

[10] [S.E.Grigorescu.2002],

N.Petkov,

and
P.Kruizinga,"Comparison of texture features based on Gabor filters", IEEE Trans. Image Processing, vol. 11,pp.1160-1167,2002.

[11] [A.K.Jain.1991], F.Farrokhnia,’Unsupervised texture segmentation using Gabor filters", Pattern Recognition, Vol.24,n.12,pp.1167-1186,1991.

[12] [J.Zhang.2002], T.Tan, «Brief review of invariant texture analysis methods », Pattern Recognition, Vol.35,pp.735-747,2002. 